\title{
Warping in the valence band of silicon
}

\author{
D. Helmholz* and L. C. Lew Yan Voon \\ Department of Physics, Worcester Polytechnic Institute, 100 Institute Road, Worcester, Massachusetts 01609
}

(Received 20 December 2001; revised manuscript received 05 March 2002; published 3 June 2002)

\begin{abstract}
The origin of warping in the valence band of silicon is studied using tight-binding and $k \cdot p$ calculations. A number of new analytical expressions for the dispersion and effective masses are given. A measure of warping is also proposed.
\end{abstract}

DOI: 10.1103/PhysRevB.65.233204

\section{INTRODUCTION}

The study of the valence band (VB) of diamond-type semiconductors goes back at least as far back as a paper by Shockley. ${ }^{1}$ Detailed study commenced in the classic paper by Dresselhaus, Kip, and Kittel ${ }^{2}$ in which the $A, B, C$ parameters were introduced in a three-band $k \cdot p$ model to describe the structure of the VB; the parameters were determined from measurements of the effective mass as a function of angle in $k$ space in cyclotron experiments. The modern importance of a detailed understanding of the VB rests upon its role in heterostructure physics; as a result, newer studies have emerged. One such example is the attempt to reproduce the warping using tight-binding (TB) models. ${ }^{3}$ In the latter paper, Boykin et al. derived a number of interesting constraints that must be satisfied by the VB effective masses for zinc-blende semiconductors. They then used measured warping, i.e., masses differing along different $k$ directions, to propose obtaining better TB parameter sets.

The purpose of the present Brief Report is to study how well TB models can reproduce warping. Our goal is not primarily to obtain better parameter sets, as in Boykin et al., ${ }^{3}$ but to see what kind of a TB model is needed in order to reproduce warping. Our goal was also prompted by a statement by Fishman ${ }^{4}$ that $s p^{3}$ TB models reproduce isotropic VB effective masses.

The Brief Report is organized as follows. We first present some new results for the exact energy dispersions of the heavy-hole $(h h)$ and light-hole $(l h)$ states and of the corresponding effective masses along certain directions in $k$ space using a third-nearest-neighbor ( $3 \mathrm{NN}) s p^{3}$ model without spin-orbit coupling (no spin). We have then computed the
PACS number(s): 71.15. $-\mathrm{m}$

valence-band dispersion of silicon using a number of different TB parameter sets and models. However, detailed results will only be presented for the three parameter sets which reproduce very accurate overall band structures. ${ }^{5-7}$

\section{VALENCE BAND OF DIAMOND-TYPE SEMICONDUCTORS}

The existence of exact analytic expressions for energy dispersions depend upon not only the structureunder study but also the model used. For example, a three-band $k \cdot p$ model for diamond has been known to give the VB energies for an arbitrary wave vector: ${ }^{2}$

$$
E=A k^{2} \pm \sqrt{B^{2} k^{4}+C^{2}\left(k_{x}^{2} k_{y}^{2}+k_{y}^{2} k_{z}^{2}+k_{x}^{2} k_{z}^{2}\right)} .
$$

No analogous exact dispersion relation for valence states for arbitrary wave vectors can be obtained even within nearest-neighbor TB theory. Slater and $\operatorname{Koster}^{8}$ pointed out that exact dispersions can be obtained for the $\Delta$ direction and for the $h h$ states along the $\Lambda$ direction, using an eight-band $s p^{3}$ model; however, they did not give those expressions. More recently, Loehr and Talwar ${ }^{9}$ gave some dispersions and effective masses along [100] for a second-nearest-neighbor $(2 \mathrm{NN}) s p^{3}$ no-spin model; Boykin and co-workers have also obtained [100] masses for the valence band at the $\Gamma$ point using a $2 \mathrm{NN} s p^{3} s^{*}$ spin-orbit model. ${ }^{10,11}$ Boykin et al. ${ }^{3}$ also gave masses along [110] and [111] using a $2 \mathrm{NN} s p^{3}$ model with spin. All of the above work was for zinc-blende crystals.

We here give the equations that are obtained using a thirdnearest-neighbor $s p^{3}$ model for diamond crystals (in the absence of spin):

$$
\begin{aligned}
E_{[100]}^{h h}= & E_{p}+W_{x x}(110)+\left[W_{x x}(011)+W_{x x}(110)\right] \cos \frac{k a_{0}}{2}-\left[\left(V_{x x}+U_{x x}(311)+2 U_{x x}(113) \cos \frac{k a_{0}}{2}\right)^{2} \cos ^{2} \frac{k a_{0}}{4}\right. \\
& \left.+\left(U_{x y}(113) \sin \frac{3 k a_{0}}{4}-\left[V_{x y}-U_{x y}(131)-U_{x y}(311)\right] \sin \frac{k a_{0}}{4}\right)^{2}\right]^{1 / 2}, \\
E_{[100]}^{l h}= & \frac{1}{2}\left\{E_{s}+E_{p}+W_{s s}(110)+W_{x x}(011)-\left[V_{s s}+V_{x x}+2 U_{s s}(311)+2 U_{x x}(113)\right] \cos \frac{k a_{0}}{4}+2\left[W_{s s}(110)\right.\right. \\
& \left.+W_{x x}(110)\right] \cos \frac{k a_{0}}{2}-\left[U_{s s}(311)+U_{x x}(311)\right] \cos \frac{3 k a_{0}}{4} \\
& -\left[\left(E_{s}-E_{p}+W_{s s}(110)-W_{x x}(011)+\left[V_{x x}-V_{s s}+2 U_{x x}(113)-2 U_{s s}(311)\right] \cos \frac{k a_{0}}{4}\right.\right.
\end{aligned}
$$




$$
\begin{aligned}
+ & 2\left[W_{s s}(110)-W_{x x}(110)\right] \cos \frac{k a_{0}}{2}-\left[U_{s s}(311)-U_{x x}(311)\right] \cos \frac{3 k a_{0}}{4} \\
+ & 4\left(V_{s x}-4 W_{s x}(110) \cos \frac{k a_{0}}{4}+2 U_{s x}(311) \cos \frac{k a_{0}}{2}+2 U_{s x}(113)+U_{s x}(311)\right)^{2} \sin ^{2} \frac{k a_{0}}{4} 1 / 2, \\
E_{[111]}^{h h}= & E_{p}+\left[W_{x x}(011)+2 W_{x x}(110)\right] \cos ^{2} \frac{k a_{0}}{2}+W_{x y}(110) \sin ^{2} \frac{k a_{0}}{2}-\frac{1}{4}\left[4 \operatorname { c o s } ^ { 2 } \frac { k a _ { 0 } } { 4 } \left(V_{x x}+V_{x y}-2 U_{x y}(113)+\left[V_{x x}-V_{x y}\right.\right.\right. \\
& \left.+2 U_{x x}(113)+U_{x x}(311)+3 U_{x y}(113)+U_{x y}(131)+U_{x y}(311)\right] \cos \frac{k a_{0}}{2}+\left[2 U_{x x}(113)+U_{x x}(311)-U_{x y}(113)\right. \\
& \left.\left.-U_{x y}(131)-U_{x y}(311)\right] \cos k a_{0}\right)^{2}+\left(\left[-3 V_{x x}-V_{x y}+U_{x y}(113)-2 U_{x x}(113)-U_{x x}(311)-U_{x y}(131)-U_{x y}(311)\right]\right. \\
& \times \sin \frac{k a_{0}}{4}+\left[V_{x x}-V_{x y}+4 U_{x x}(113)+2 U_{x x}(311)+2 U_{x y}(113)\right] \sin \frac{3 k a_{0}}{4}+\left[U_{x y}(113)-2 U_{x x}(113)-U_{x x}(311)\right. \\
& \left.\left.\left.+U_{x y}(131)+U_{x y}(311)\right] \sin \frac{5 k a_{0}}{4}\right)^{2}\right],
\end{aligned}
$$

where the E's, $V$ 's, $W$ 's, and $U$ 's are on-site, nearestneighbor, second-nearest-neighbor, and third-nearestneighbor TB parameters, respectively (see Ref. 12 for exact definitions), $a_{0}$ is the lattice constant, and $k$ is the magnitude of the wave vector in the respective directions.

We have also obtained a number of effective masses exactly for the $3 \mathrm{NN}$ no-spin $s p^{3}$ model:

$$
\begin{aligned}
& \frac{1}{m_{[100]}^{h h}}=\frac{a_{0}^{2}}{16 \hbar^{2}}\left[V_{x x}-4 W_{x x}(011)-4 W_{x x}(110)\right. \\
& +10 U_{x x}(113)+U_{x x}(311) \\
& \left.-\frac{\left[V_{x y}-3 U_{x y}(113)-U_{x y}(131)-U_{x y}(311)\right]^{2}}{V_{x x}+2 U_{x x}(113)+U_{x x}(311)}\right] \text {, } \\
& \frac{1}{m_{[100]}^{l h}}=\frac{a_{0}^{2}}{16 \hbar^{2}}\left[V_{x x}-8 W_{x x}(110)+2 U_{x x}(113)+9 U_{x x}(311)\right. \\
& -2\left[2 U_{s x}(113)+3 U_{s x}(311)+V_{s x}-4 W_{s x}(110)\right]^{2} \\
& \times\left[E_{s}-E_{p}-V_{s s}+V_{x x}+3 W_{s s}(110)-W_{x x}(011)\right. \\
& -2 W_{x x}(110)-3 U_{s s}(311)+2 U_{x x}(113) \\
& \left.\left.+U_{x x}(311)\right]^{-1}\right] \\
& \frac{1}{m_{[111]}^{h h}}=\frac{a_{0}^{2}}{16 \hbar^{2}}\left[3 V_{x x}-2 V_{x y}-8 W_{x x}(011)-16 W_{x x}(110)\right. \\
& +8 W_{x y}(110)+22 U_{x x}(113)+11 U_{x x}(311) \\
& -2 U_{x y}(113)-6 U_{x y}(131)-6 U_{x y}(311) \\
& \left.-\frac{\left[V_{x y}-3 U_{x y}(113)-U_{x y}(131)-U_{x y}(311)\right]^{2}}{V_{x x}+2 U_{x x}(113)+U_{x x}(311)}\right] \text {. }
\end{aligned}
$$

The latter equations can be used to evaluate the effective masses exactly, without recourse to numerical differentiation. (Note, however, that another general approach is given in Ref. 13.) This is advantageous when implementing fitting algorithms. The relation among effective masses obtained by Boykin et $a l .{ }^{3}$ can be trivially obtained from a $k \cdot p$ model; we have verified this using Eq. (1). Hence, that result, derived using a $2 \mathrm{NN}$ TB model in Ref. 3, is a general one. Furthermore, we will be deriving the $k \cdot p$ parameters from TB models. For this, we will use the following equations, again derived from Eq. (1):

$$
\begin{gathered}
A=\frac{\hbar^{2}}{4}\left(\frac{1}{m_{[100]}^{h h}}+\frac{1}{m_{[100]}^{l h}}\right), \\
|B|=\frac{\hbar^{2}}{4}\left(\frac{1}{m_{[100]}^{h h}}-\frac{1}{m_{[100]}^{l h}}\right), \\
|C|=\frac{\hbar^{2}}{4} \\
\times \sqrt{3\left[\left(\frac{2}{m_{[111]}^{h h}}-\frac{1}{m_{[100]}^{h h}}-\frac{1}{m_{[100]}^{l h}}\right)^{2}-\left(\frac{1}{m_{[100]}^{h h}}-\frac{1}{m_{[100]}^{l h}}\right)^{2}\right]} .
\end{gathered}
$$

Note that the only physical solution with $C=0$ is given by $m_{[111]}^{h h}=m_{[100]}^{h h}$, as it should. One can now insert Eqs. (5)-(7) into Eqs. (8) to express the $k \cdot p A, B, C$ parameters in terms of TB ones. The expressions for the $3 \mathrm{NN}$ TB model are too lengthy to warrant reproducing here. For the 1NN TB model, one gets

$$
\begin{gathered}
A=\frac{a_{0}^{2}}{64}\left[2 V_{x x}-\frac{V_{x y}^{2}}{V_{x x}}-\frac{2 V_{s x}^{2}}{E_{s}-E_{p}-V_{s s}+V_{x x}}\right], \\
|B|=\frac{a_{0}^{2}}{64}\left[\frac{2 V_{s x}^{2}}{E_{s}-E_{p}-V_{s s}+V_{x x}}-\frac{V_{x y}^{2}}{V_{x x}}\right],
\end{gathered}
$$




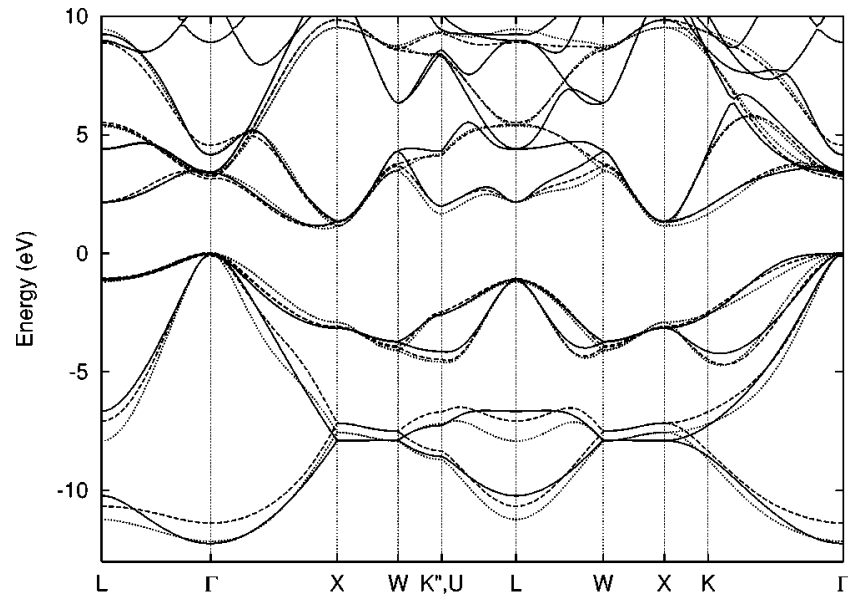

FIG. 1. Band structure of Si calculated with the TB parameters of Jancu et al. (Ref. 6) (solid lines), Tserbak et al. (Ref. 5) (dotted lines), and Niquet et al. (Ref. 7) (dashed lines). All three models include spin-orbit coupling.

$$
\begin{aligned}
|C|= & \frac{\sqrt{3} a_{0}^{2}}{16}\left[2\left(V_{x x}-V_{x y}\right)^{2}\right. \\
& \left.-\left(V_{x x}-V_{x y}\right)\left(\frac{V_{x y}^{2}}{V_{x x}}+\frac{2 V_{s x}^{2}}{E_{s}-E_{p}-V_{s s}+V_{x x}}\right)\right]^{1 / 2} .
\end{aligned}
$$

This approach for relating a TB model to a $k \cdot p$ one does not appear to have been used before. One finds the interesting result that, for a $1 \mathrm{NN} s p^{3}$ no-spin model, the warping would be exactly zero if $V_{x x}=V_{x y}$. This condition is relaxed if one introduces $d$ orbitals or if one goes beyond 1NN. These two cases will be studied numerically in the next section.

\section{COMPARISONS}

We have recalculated the band structure along various directions using the TB models of Tserbak et al., ${ }^{5}$ Jancu et al., ${ }^{6}$ and Niquet et al. ${ }^{7}$ Figure 1 shows that they are in very good overall agreement. We remind the reader that all three models include spin-orbit coupling.

Next, we compare the warping that is obtained using these models and using a three-band $k \cdot p$ model. We show, in Fig. 2(a), the dispersions when going from $\Delta$ to $\Sigma$ to $\Lambda$ for fixed $k=0.02 \pi / a_{0}$ using the three models. We see that all three models display warping. Indeed, the calculated effective masses are all anisotropic (Table I). This is in contrast to what was claimed by Fishman. The reason is because the model used by Fishman is actually a bond-orbital model with only two parameters. Indeed, it is a simplified version of a TB model; for example, such a model has a dispersionless $h h$ band. Figure 2(a) shows that the $s p^{3} d^{5} s^{*}$ model is closer to $k \cdot p$ than the $s p^{3}$ model of Tserbak et al. (which underestimates warping) but not when compared to the $s p^{3}$ model of Niquet et al. (see also Table I). Defining a measure of the warping to be given by

$$
\delta(\Sigma, \Delta) \equiv \lim _{k \rightarrow 0} \frac{E(\Delta)-E(\Sigma)}{E(\Delta)},
$$
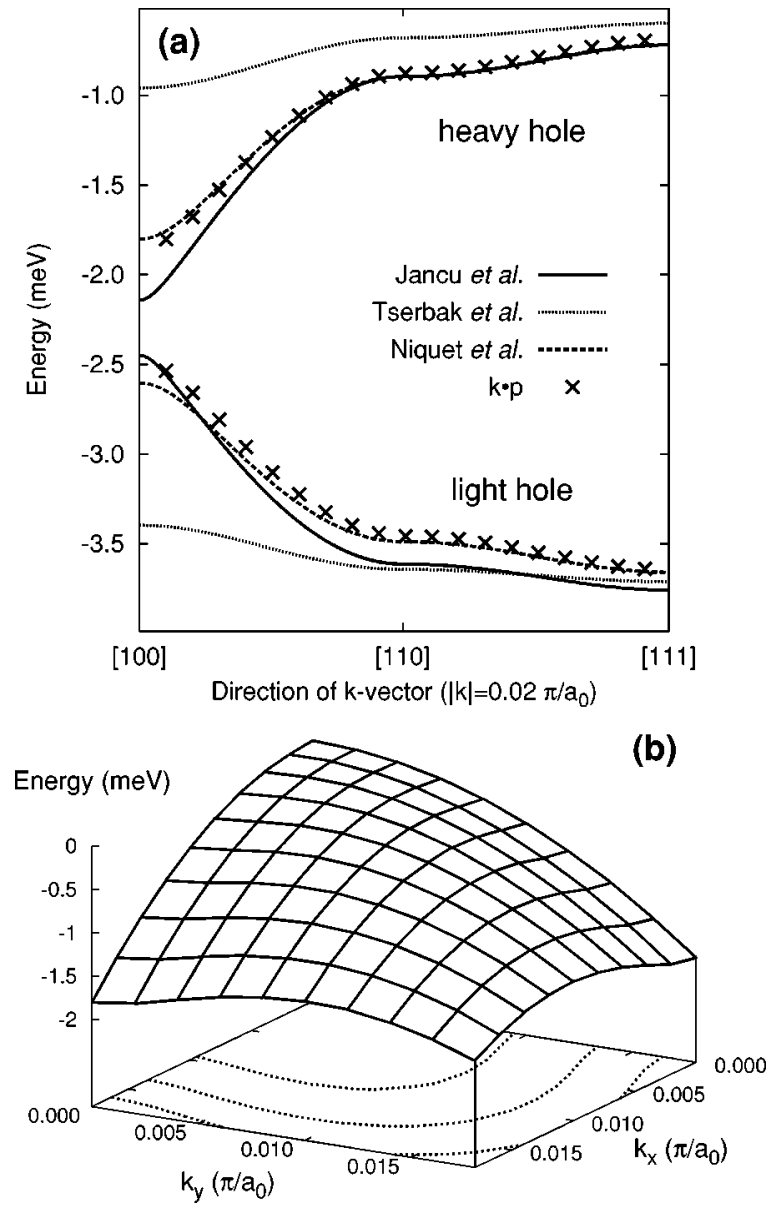

FIG. 2. (a) Energy dispersion from $\Delta$ to $\Sigma$ to $\Lambda$ for $k$ $=0.02 \pi / a_{0}$ of the $h h$ and $l h$ bands calculated using a $k \cdot p$ model (Ref. 15) (crosses), TB models from Jancu et al. (Ref. 6) (solid lines), Tserbak et al. (Ref. 5) (dotted line), and Niquet et al. (Ref. 7) (dashed line). (b) Representation of warping for Niquet et al. (Ref. $7)$. The contour lines are for energies of $-0.5,-1.0$, and $-1.5 \mathrm{meV}$.

one finds that the $h h$ warping is 0.53 for $k \cdot p, 0.57$ for Jancu et al., 0.29 for Tserbak et al., and 0.50 for Niquet et al. Similar results are obtained if one calculates $\delta(\Lambda, \Delta)$ rather than $\delta(\Sigma, \Delta)$. That $\delta(\Sigma, \Delta)$ provides a good measure of warping can be seen by calculating the corresponding $A B C$ parameters for the three TB models using Eq. (8) (Table II). The

TABLE I. $h h$ and $l h$ effective masses (in units of electron rest mass) along different directions, calculated using $k \cdot p$ [parameters fitted to experimental data (Ref. 15)] and three TB models.

\begin{tabular}{lcccc}
\hline \hline & & Jancu et al. & Tserbak et al. & Niquet et al. \\
& $k \cdot p$ & $s p^{3} d^{5} s^{*}$ & $s p^{3}$ & $s p^{3}$ \\
\hline$m_{[100]}^{h h}$ & -0.275 & -0.238 & -0.532 & -0.283 \\
$m_{[110]}^{h h}$ & -0.580 & -0.540 & -0.740 & -0.567 \\
$m_{[111]}^{h h}$ & -0.738 & -0.710 & -0.853 & -0.712 \\
$m_{[100]}^{l h}$ & -0.204 & -0.208 & -0.147 & -0.200 \\
$m_{[110]}^{l h}$ & -0.147 & -0.139 & -0.136 & -0.145 \\
$m_{[111]}^{l h}$ & -0.139 & -0.132 & -0.133 & -0.138 \\
\hline \hline
\end{tabular}


TABLE II. $k \cdot p$ parameters, from Ref. 15 for $k \cdot p$ and calculated from effective masses for TB.

\begin{tabular}{lcccc}
\hline \hline & & $\begin{array}{c}\text { Jancu } \text { et al. } \\
s p^{3} d^{5} s^{*}\end{array}$ & $\begin{array}{c}\text { Tserbak et al. } \\
s p^{3}\end{array}$ & $\begin{array}{c}\text { Niquet } \text { et al. } \\
s p^{3}\end{array}$ \\
\hline$A$ & -4.27 & -4.50 & -4.34 & -4.32 \\
$|B|$ & 0.63 & 0.30 & 2.46 & 0.79 \\
$|C|$ & 4.93 & 5.34 & 3.46 & 4.99 \\
\hline \hline
\end{tabular}

same trend in warping is found. In Fig. 2(b), we provide more conventional plots of the warping obtained using $\mathrm{Ni}$ quet et al.

Similar calculations of warping using a number of other $s p^{3}$ TB parameter sets that do not reproduce the overall band structure as well led to poorly reproduced warping as well. One concludes that $d$ orbitals are not required to generate warping per se or to generate substantial warping. Indeed, the fact that an $s p^{3}$ model can reproduce warping similar to that obtained from $k \cdot p$ theory is implicit in the paper by Chang ${ }^{14}$ where a three-band $s p$-based effective bond-orbital model (EBOM) was equated to the $k \cdot p$ Hamiltonian. Obviously, one advantage of all the localized-orbital models (TB, $\mathrm{EBOM})$ is that they display nonparabolicity of the valence bands.

We have next compared the warping of the $l h$ band to that of the $h h$ band. For $l h$, our measure of warping gives -0.38 for $k \cdot p,-0.50$ for Jancu et al., -0.08 for Tserbak et al., and -0.35 for Niquet et al. We note that in $k \cdot p$ theory, a single parameter $(C)$ is used to characterize the warping, even though the warping is known to be different for the $h h$ and $l h$ bands. Thus, it is often said that there is no warping if $C=0$. While this is certainly true, $C$ alone does not provide a measure of the difference in warping between the $h h$ and $l h$ bands. This is evident if one derives the $k \cdot p$ representation of our warping parameter:

$$
\delta_{h h, l h}(\Sigma, \Delta)=1-\frac{A \pm \sqrt{B^{2}+\frac{C^{2}}{4}}}{A \pm|B|} .
$$

In addition, combining Eqs. (8) and (11), one can write $\delta_{h h, l h}(\Sigma, \Delta)$ solely in terms of effective masses. This provides an alternative way from Eq. (10) of obtaining the new
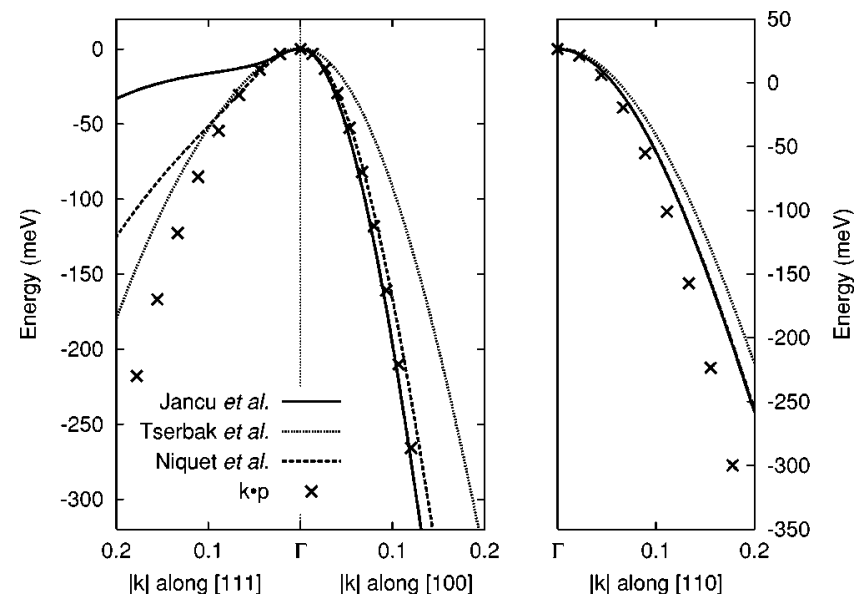

FIG. 3. Energy dispersion along three directions.

warping parameters that neither requires obtaining the dispersions nor is dependent upon a specific $k \cdot p$ model.

For completeness, we have also calculated the nonparabolicity in the valence band. We found the nonparabolicity of the $l h$ band to be small for all the TB models. For the $h h$ band, Niquet et al. and Jancu et al. give very similar dispersions except for [111] where the $s p^{3} d^{5} s^{*}$ model displays significantly larger nonparabolicity (Fig. 3). This demonstrates that warping and nonparabolicity are not related. Further study of the role of nonparabolicity would require a more accurate knowledge within $k \cdot p$ theory (it is, of course, absent within the current three-band model).

In summary, we have proposed a simple parameter to distinguish the warping of the valence band due to different bands. We have also derived a number of new exact dispersions and effective masses within TB theory and, also, new equations connecting TB and $k \cdot p$ theories. We have shown that $d$ orbitals are not required to reproduce significant warping of the valence bands of diamond-type semiconductors but can, nevertheless, do so. Finally it is also suggested that nonparabolicity be used as an additional way of ascertaining how good a TB parameter set is.

\section{ACKNOWLEDGMENTS}

This work was supported by an NSF CAREER award (NSF Grant No. 9984059). D.H. would like to thank Professor W.-K. Ge for financial support.
* On leave from The Hong Kong University of Science and Technology, Department of Physics, Clear Water Bay, Hong Kong.

${ }^{1}$ W. Shockley, Phys. Rev. 90, 491 (1953).

${ }^{2}$ G. Dresselhaus, A. Kip, and C. Kittel, Phys. Rev. 98, 368 (1955).

${ }^{3}$ T.B. Boykin, L.J. Gamble, G. Klimeck, and R.C. Bowen, Phys. Rev. B 59, 7301 (1999).

${ }^{4} \mathrm{G}$. Fishman, Energie et fonction d'onde des semi-conducteurs (Les Editions de Physique, Paris, 1988), p. 29.

${ }^{5}$ C. Tserbak, H.M. Polatoglou, and G. Theodorou, Phys. Rev. B 47, 7104 (1993).

${ }^{6}$ J.M. Jancu, R. Scholz, F. Beltram, and F. Bassani, Phys. Rev. B 57, 6493 (1998).
${ }^{7}$ Y.M. Niquet, C. Delerue, G. Allan, and M. Lannoo, Phys. Rev. B 62, 5109 (2000).

${ }^{8}$ J. Slater and G. Koster, Phys. Rev. 94, 1498 (1954).

${ }^{9}$ J.P. Loehr and D.N. Talwar, Phys. Rev. B 55, 4353 (1997).

${ }^{10}$ T.B. Boykin, G. Klimeck, R.C. Bowen, and R. Lake, Phys. Rev. B 56, 4102 (1997).

${ }^{11}$ T.B. Boykin, Phys. Rev. B 56, 9613 (1997).

${ }^{12}$ L. C. Lew Yan Voon, Ph.D. thesis, Worcester Polytechnic Institute, Worcester, MA, 1993.

${ }^{13}$ T.B. Boykin, Phys. Rev. 52, 16317 (1995).

${ }^{14}$ Y.C. Chang, Phys. Rev. B 37, 8215 (1988).

${ }^{15}$ Semiconductors: Group IV Elements and III-V Compounds, edited by O. Madelung (Springer-Verlag, Berlin, New York, 1991). 